\title{
Evaluación de la Consejería Pretest para el VIH en un Hospital Nacional en Lima-Perú
}

\author{
Evaluating HIV pre-test counseling in people attending a national \\ hospital in Lima, Peru
}

Otto E. Mora-Salazar' ${ }^{1}$ Jorge D. Palomino-Vargas ${ }^{1}$, Cecilia M. Velásquez-Ruiz ${ }^{1}$, Enrique M. Gómez-Pomar ${ }^{1}$, Rosa M. Castillo-Schilder ${ }^{1}$, Leslie Soto-Arquiñigo ${ }^{2}$ y Frine Samalvides-Cuba ${ }^{2}$

1 Universidad Peruana Cayetano Heredia. Lima, Perú. ottomora@hotmail.com, coqui_palomino@hotmail.com, cecy_med@hotmail.com, egomezpomar@gmail.com, castillo.roma@gmail.com

2 Hospital Nacional Cayetano Heredia. Lima, Perú. les_soto@hotmail.com, frine@upch.edu.pe

Recibido 16 Diciembre 2008/Enviado para Modificación 6 Julio 2009/Aceptado 18 Julio 2009

\section{RESUMEN}

Objetivo Evaluar los conocimientos adquiridos por la consejería pretest para el VIH, en personas que acudieron a un hospital nacional de Lima, Perú.

Método Estudio observacional descriptivo de corte transversal, que se realizó en 499 personas que acudieron a la Estrategia Sanitaria Nacional de Prevención y Control de ITS/VIH y SIDA, que fueron seleccionadas de manera probabilística de tipo intencional. Se aplicó una entrevista estructurada que utilizó un cuestionario validado por expertos con preguntas directas. Se definió como variable de conocimiento correcto a un puntaje $\geq 75 \%$ de respuestas correctas y como variable de conocimiento incorrecto a un puntaje $<75 \%$ de respuestas incorrectas.

Resultados El 64,1\% $(n=320)$ de los encuestados fueron mujeres. El grupo de edad con mayor numero de respuestas correctas $(40,3 \%, n=201)$ fue el de 22 a 28 años, $(p<0,05)$. Las personas que acudieron por pareja con diagnóstico reciente de $\mathrm{VIH} /$ SIDA, tuvieron mayor cantidad de respuestas correctas (12,3 en promedio). Los hombres alcanzaron un mayor nivel de conocimientos correctos $(63,6 \%)$ en comparación con las mujeres $(36,4 \%),(p<0,05)$. Solo 4,4\% $(n=22)$ del total, alcanzaron un nivel de conocimiento correcto.

Conclusión El nivel de conocimiento adquirido con la consejería pretest en este hospital fue deficiente, no cumpliendo los objetivos de la misma. Se sugiere cambiar la metodología para que pueda aumentar la retención de información por las personas, y de esta manera reducir la morbi-mortalidad en ITS y VIH/ SIDA.

Palabras Clave: Test, VIH, conocimiento, evaluación (fuente: DeCS, BIREME).

\section{ABSTRACT}

Objective Evaluating the knowledge acquired by the pre-test counselling programme in a national hospital in Lima Peru. 
Method A cross-sectional descriptive observational study was carried out on 499 patients attending the National STI/HIV Prevention and Control Sanitary Strategy service after they had been counselled. The patients were intentionally selected by probabilistic means. A questionnaire having direct questions was applied; it had been previously validated by experts. $\geq 75 \%$ of correct answers was defined as being a variable of correct knowledge and $<75 \%$ of correct answers as incorrect knowledge.

Results $64.1 \%(n=320)$ of the patients were female. The age group having a better level of knowledge after counselling was the 22-28 year old group, $40.3 \%(n=201)$ $(p<0.05)$. People having a partner diagnosed as having HIV/AIDS answered more questions correctly (12.3 on average). Men showed higher correct knowledge (63.6\%) related to women $(36.4 \%)(p<0.05)$. Only $4.4 \%(n=22)$ of the patients had a correct level of knowledge.

Conclusion The pre-test counselling programme did not improve knowledge regarding HIV/AIDS. It is thus suggested that the approach and method should be changed so that patients become well-informed, thereby reducing STI and HIV/AIDS-related morbidity and mortality.

Key Words: Counselling, HIV, knowledge, test (source: MeSH, NLM).

$\mathrm{L}$ a infección por el virus de inmunodeficiencia humana (VIH) es una pandemia mundial que afecta sin distinción de condición económica, edad, sexo o raza, con un estimado de 33,2 millones de personas viviendo con VIH/SIDA (síndrome de inmunodeficiencia adquirida) en el 2007. La incidencia en el Perú es de 20610 casos de SIDA y 30389 de infección por VIH. (1)

La consejería pre test es un instrumento que contribuye, teóricamente, desde el punto de vista epidemiológico a romper la cadena de transmisión del VIH, al suministrar información veraz que favorece la reflexión de las personas sobre sus riesgos de infección, permite evaluar la percepción de los mismos, desarrollar acciones preventivas y promover estilos de vida saludables, así como identificar los riesgos a los que están expuestos. Así mismo, esclarece creencias equívocas o fantasías que aún persisten con respecto a la infección, disminuye el estrés en la espera del resultado y promueve la adherencia al tratamiento, por lo que mejora la calidad de vida de las personas (2).

Las pruebas para diagnosticar la infección por el VIH son voluntarias en el Perú y se realizan previa consejería obligatoria según el artículo 2 de la Ley $\mathrm{N}^{\circ} 28$ 243. (3) Un aspecto importante de esta consejería es obtener consentimiento informado para llevar a cabo el examen y conocer los resultados. (4)

Mediante el presente trabajo, se evaluaron los conocimientos adquiridos por las personas mediante la consejería previa a la toma del test para la detección de infección por el VIH. 


\section{MATERIALES Y MÉTODOS}

Se realizó un estudio descriptivo transversal en donde se utilizó una encuesta anónima, basada en el manual utilizado para la consejería brindada por el Programa de Control de Enfermedades de Transmisión Sexual y SIDA (PROCETSS), ahora denominado Estrategia Sanitaria Nacional de Prevención y Control de ITS y VIH/SIDA. La encuesta se realizo a las personas que acudieron a la toma de muestra para el diagnostico de la infección por el VIH, y posterior a haber recibido la consejería respectiva. Debe señalarse que en el Perú, es obligatoria la consejería sobre VIH e ITS a toda persona previa a la prueba de Elisa para el VIH.

Con un promedio de población que recibe consejería pretest/año de 4800 personas, se calculó el tamaño de la muestra con el programa EPI INFO versión 3.3.2 con un nivel de confianza del $99 \%$, un error del $5 \%$, una frecuencia esperada de entendimiento de $30 \%$ y un peor resultado esperado de $25 \%$. La muestra obtenida fue de 499 pacientes que se eligieron de manera aleatoria y que cumplieron con los criterios de inclusión.

Los criterios de inclusión admitían a toda persona mayor de edad que haya recibido la consejería en VIH y SIDA en el PROCETSS. Los criterios de exclusión a su vez, descartaron a personas que hubiesen recibido la consejería en otro centro de salud, que no la hubiesen recibido, a las que presentaban alteraciones mentales y a las que ya estaban viviendo con el VIH/SIDA.

Se definió como variable de conocimiento correcto un puntaje igual o superior al $75 \%$ de respuestas respondidas correctamente y como variable de conocimiento incorrecto a un puntaje inferior al $75 \%$ de respuestas respondidas correctamente.

La encuesta consta de cuatro partes: Primera parte: datos generales como son: edad, sexo, fecha de consejería, etc. Segunda parte: evaluación del nivel de conocimientos generales con respecto al VIH/SIDA, mediante 25 preguntas dicotómicas. Tercera parte: constituida por 5 preguntas con los cuales se recogió información sobre embarazo e infección por VIH. Cuarta parte: se evaluó la ley 26626 "contra SIDA".

Se protegió la confidencialidad de los encuestados y el consentimiento informado fue la aceptación de las personas a responder la encuesta. 
La encuesta fue previamente validada por expertos en una población piloto de 50 personas que cumplían los criterios de inclusión. Se corrigieron los ítems de practicidad, comprensión y redacción de la encuesta obteniéndose la versión final. Se consideró los principios de anonimato, libre participación y confidencialidad de las personas que participaron en este estudio.

Los datos recolectados en la encuesta se procesaron en el paquete estadístico SPSS. Las variables categóricas se presentaron en tablas de frecuencias y porcentajes; las variables numéricas con medidas de resumen y dispersión (medias y desviación estándar). En el análisis bivariado se aplicó las pruebas noparamétricas de Chi-cuadrado (variables categóricas) y la de U de Mann-Whitney (variables numéricas). También se utilizó la Prueba no-paramétrica U de MannWhitney, adecuada cuando los valores de la variable no siguen una distribución normal. La Prueba de Chi-cuadrado se aplicó a tablas de contingencia para determinar la asociación entre dos variables categóricas. En todas las pruebas se utilizó un nivel de significancia de 0,05 .

\section{RESULTADOS}

Se aplicó la encuesta a 499 personas que cumplieron con los criterios establecidos, de las cuales el $64,1 \%(n=320)$ eran mujeres y el 35,9\% $(n=179)$ varones.

Los motivos de consulta por consejería se indican en la Tabla 1.

Tabla 1. Motivo de la consulta por consejería en la población estudiada

\begin{tabular}{lcc}
\hline \multicolumn{1}{c}{ Motivo Consejería } & No. & $\%$ \\
\hline Gestación & 165 & 33,1 \\
Sospecha de infección por VIH & 164 & 32,9 \\
Pareja con diagnostico reciente de VIH/SIDA & 62 & 12,4 \\
Requisito pre-Matrimonial & 34 & 6,8 \\
Requisito para Riesgo Quirúrgico & 63 & 12,6 \\
Abuso sexual & 11 & 2,2 \\
\hline
\end{tabular}

El grupo de edad con mayor numero de respuestas correctas fueron los de 22 a 28 años $(p<0,01)$, como se indica en la Figura 1. Los que menos respondieron correctamente fueron los más jóvenes (menor o igual a 21 años) y los de mayor edad (43 o más años) (Figura 2).

Se encontró que los hombres alcanzaron una mayor proporción de espuestas correctas con respecto a las mujeres, 64 vs. $36 \%$. Así mismo ocurrió con los promedios $(10,7 \pm 4,9$ preguntas correctas vs. $9,5 \pm 4,5$ preguntas correctas) $(\mathrm{p}<0,05)$. 
Figura 1. Distribución de las personas por grupo etáreo

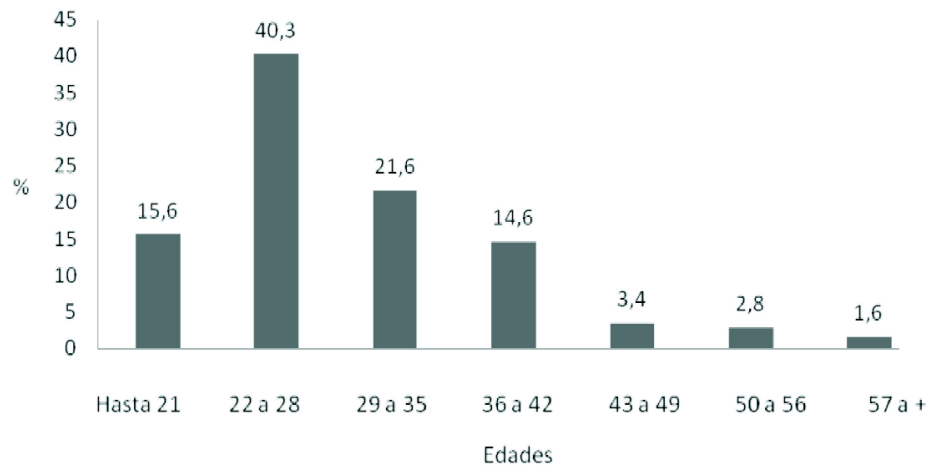

Figura 2. Distribución de conocimiento correcto o incorrecto de acuerdo a grupo etáreo

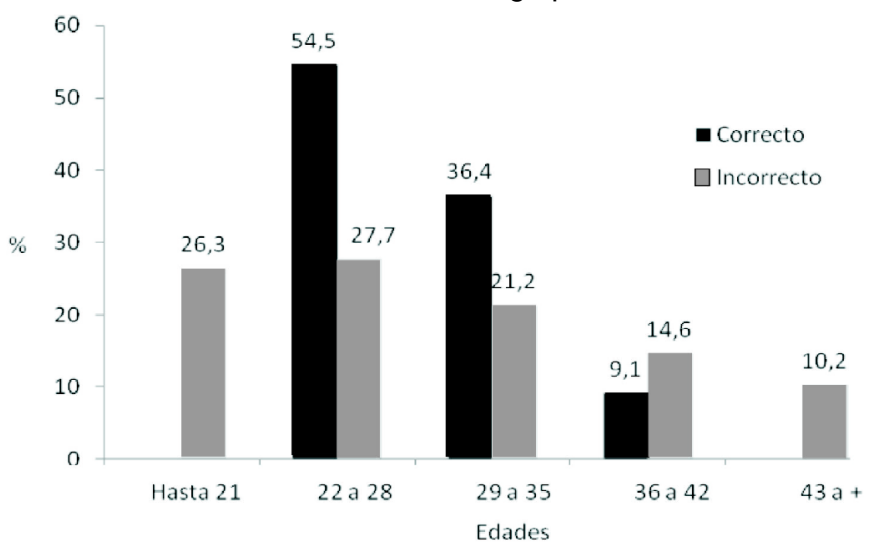

En conocimientos se observa que los que acudían a tomarse el test diagnostico por tener una pareja con diagnóstico reciente de VIH/SIDA, fueron los que obtuvieron mayor numero de respuestas correctas $(12,3 / 35$ preguntas correctas en promedio) y los que menos respondieron correctamente fueron los que acudían como parte de la evaluación de riesgo quirúrgico $(8,5 / 35$ preguntas incorrectas en promedio).

Se obtuvo que solo el 4,4\% $(\mathrm{n}=22)$ de las personas alcanzaron un conocimiento correcto, de estos el 45,5\% (n=10) fue el grupo con sospecha de infección por VIH. El 95,6 \% $(n=477)$ de personas no alcanzaron un conocimiento correcto, de estos el mayor grupo fueron las gestantes, $34 \%(n=162)$. 


\section{DISCUSIÓN}

La Consejería pretest es un instrumento importante para dar información veraz y precisa sobre las ITS y el VIH/SIDA (5). Es de esperarse que la mayoría de las personas que la reciben muestren un alto nivel de información y retengan el conocimiento presentado. De esta manera se podría disminuir la morbimortalidad que estas enfermedades conllevan (6).

En relación con las edades, se encontró que el grupo de 22 a 28 años fue el que más acudió a recibir consejería $(40,3 \% \mathrm{n}=201)$ (Figura 1). Esto coincide con las edades en que es más probable contraer las ITS, lo que hace pensar que acuden más las personas para quienes las ITS o el VIH/SIDA constituyen un problema, ya sea porque se perciben en riesgo o porque ya han sido diagnosticadas con alguna ITS (7). Por el contrario los encuestados más jóvenes asimilaron muy poca información. Esto podría asociarse a que muchos jóvenes puedan percibir la posibilidad de infectarse con el VIH como algo ajeno a ellos.

Respecto al sexo de las personas que acuden a la consejería, se aprecia una diferencia significativa de casi el doble de las mujeres con respecto a los hombres. Esta diferencia se explicaría por la gran cantidad de consultantes que se toman la prueba diagnóstica como parte de los exámenes pre-natales, ya que el descarte de la infección por el VIH es una prueba obligatoria en el primer trimestre del periodo gestacional (8).

Respecto al nivel de conocimiento, se observó que las personas que acudían a la consejería por tener una pareja con diagnóstico reciente de VIH/SIDA, fueron los que mejor captaron el contenido y retuvieron la información. Esto puede deberse a que siendo ya el VIH/SIDA parte de su vida diaria por el estado de salud de su pareja, tienen mayor interés en la información dada en las consejerías. Los que menos asimilaron la información brindada, fueron los que acudieron por riesgo quirúrgico. Por el contrario, este grupo se realizo la prueba diagnóstica como rutina previa a una intervención quirúrgica, sin tener en mente la posibilidad de estar infectados con el VIH.

La mayor cantidad de respuestas correctas corresponde a los temas: forma de transmisión, diagnóstico y prevención del VIH, y el menor nivel de conocimientos corresponde a los rubros: diagnóstico y transmisión de VIH al recién nacido y profilaxis de la infección por el VIH en gestantes. Esto se relaciona a la difusión acerca de la enfermedad, por medio de campañas y medios de comunicación, lo cual ha ayudado de manera positiva en el conocimiento general 
de cómo se transmite, diagnostica y previene el VIH. Sin embargo es importante tomar en cuenta también los conocimientos claros sobre VIH y embarazo en la población, en especial las gestantes, para lo cual se podrían organizar campañas de difusión como soporte a la consejería, y también implementando estos temas en las consultas de planificación familiar y control pre-natal.

Respecto al uso del preservativo y su importancia en la prevención de ITS y VIH, un 60,7\% respondió de manera correcta, de este resultado se puede inferir que es imperante realizar un afianzamiento sobre este tema en la consejería. En una investigación al respecto se encontró que en el Perú, el 50 por ciento de varones sexualmente activos y el $70 \%$ de mujeres sexualmente activas reportaron nunca haber usado un condón (11). Extrapolando estos hallazgos, se hace necesario ampliar la difusión de información especialmente a la población joven, por medio de campañas de salud donde se impartan temas respecto a la prevención de las ITS y VIH/SIDA promoviendo el uso del preservativo y conciencia responsable sobre el tema.

Es preocupante que el 95,6\% de los encuestados tuvo un conocimiento incorrecto sobre las ITS y el VIH sobretodo en el rango de edad entre 20 y 30 años que conforman el 25,7 \% de la población total en nuestro país (12). Más aun teniendo en cuenta que 47,5 \% de los encuestados presentaban alguna razón válida para hacerse la prueba, lo que nos inclinaría a pensar que tienen un mayor interés en adquirir información de la consejería.

El grupo de gestantes presentó el mayor porcentaje de preguntas con respuestas incorrectas, por lo que se necesita reforzar el tema de transmisión vertical. Así se podría cumplir con uno de los objetivos principales de la consejería, que es disminuir, por medio de la educación para la prevención, la transmisión vertical, que constituye la segunda causa de transmisión en nuestro país, reflejado en el $3 \%$ de los aproximadamente 1500 niños que vivirían con VIH/SIDA en el Perú (13).

Los resultados del presente estudio muestran que es necesario modificar la metodología de la consejería pre-test de VIH, debido a que desde 1997 no se han actualizado los manuales ni la metodología de las consejerías. Es necesario que se hagan actualizaciones de acuerdo a las nuevas estrategias educativas, los datos clínicos y epidemiológicos. Este periodo estacionario permitió solamente obtener indicadores cuantitativos, es decir número de consejerías realizadas; pero no así indicadores de corte cualitativo traducidos en el impacto de la consejería en los usuarios y el grado de efectividad en el cambio de comportamientos sexuales (14). Asimilar los conocimientos, retener la información, motivar la difusión de 
información entre pares y generar un impacto en la vida diaria de las personas, sin importar el resultado de la prueba diagnóstica, deben ser los objetivos de la renovación de la consejería. El uso de métodos audiovisuales debe ser implementado ya que ha demostrado eficacia generando motivación para el aprendizaje (10).

Referente a los consejeros, se debería mejorar en la metodología de enseñanza por un lado y por otro deberían ser evaluados periódicamente para asegurar la calidad de sus servicios y brindarles apoyo y capacitación constante. $(10,14) \mathrm{La}$ capacitación debe incluir habilidades interpersonales y de empatía para con los pacientes. Además es importante que la consejería se realice en un ambiente adecuado y privado, que permita que la persona aclare todas sus dudas sin temor a ser censurado.

Este estudio fue de tipo descriptivo por lo que consideramos que podría existir sesgo al no tener datos de los encuestados en lo que se refiere al nivel educativo alcanzado y a su nivel socioeconómico, los cuales se correlacionan con el nivel de entendimiento de las preguntas, y podría ser una limitante en nuestro estudio. El conocimiento previo (consejerías previas por riesgo quirúrgico o por gestación) no fue considerado ya que el objetivo del estudio era medir el conocimiento adquirido momentos después de la consejería. Sin embargo el conocimiento previo podría haber influenciado en que a mayor número de consejerías anteriores, habría mayor conocimiento previo en relación a las ITS y $\mathrm{VIH} / \mathrm{SIDA}$

\section{REFERENCIAS}

1. Dirección General de Epidemiología (OGE). Situación del VIH/SIDA en el Perú Boletín Epidemiológico Mensual Septiembre 2007. Disponible en: http://www.oge.sld.pe/vigilancia/vih/ Boletin 2007/diciembre.pdf. Consultado en Septiembre 2007.

2. MINSA - Guía Nacional de Consejería 2006 Disponible en: www.minsa.gob.pe/portal/ 03EstrategiasNacionales/03ESN-ITS-SIDA/Archivos/GuiaNacionallTS_Dic2006.pdf. Consultado en Septiembre 2007.

3. Ministerio de Salud Pública. Directrices básicas para la realización de consejería/orientación en VIH/Sida. Uruguay; 2006. Disponible en: www.inau.gub.uy/biblioteca/sexualidad/ UNIDAD\%20IV/PDF\%20Consejeria\%20VIH.pdf. Consultado en Agosto 2007.

4. Organización Panamericana de la Salud (OPS) Infecciones de Transmisión Sexual (ITS - VIH SIDA); 2005 Disponible en: http://www.ops.org.bo/its-vih-sida/?TE=20040628161705. Consultado en Septiembre 2007.

5. Consejería cara a cara en VIH/Sida. Experiencias en Cuba. 2005. Disponible en: http://www.sld.cu/ galerias/pdf/servicios/sida/consejeria_cara_a_cara_en_vih.pdf. Consultado en Septiembre 2007.

6. Organización Panamericana de la Salud, 2005 Disponible en: www.paho.org/Spanish/ AD/FCH/ Al/MujeryVIH.pdf. Consultado en Septiembre 2007. 
7. Análisis de la situación epidemiológica en el Perú. Lima, Perú 2006. Disponible en: http:// www.oge.sld.pe/pub asis.php. Consultado en Septiembre 2007.

8. MINSA. Plan Estratégico Multisectorial (PEM) 2007-2011 para la prevención y control de las ITS y VIH/SIDA en el Perú Disponible en: www.epiredperu.net/epired/ppts/curso_ epibas/ epibas_mh07_ppt18.pdf. Consultado en Septiembre 2007.

9. Ley Pública: No.28243. Ley que amplía y modifica la Ley 26626 sobre el VIH, SIDA y las enfermedades de transmisión sexual. Lima, Perú, mayo 31, 2004. www.cajpe.org.pe/rij/ bases/legisla/peru/proleype.HTM . Consultado en Agosto 2007.

10. Khalsa AM Preventive Counseling, screening, and therapy for the patient with newly diagnosed HIV infection. Am Fam Physician 2006 Jan 15; 73(2): 271-80.

11. Intituto Nacional de Estadística e Informática (INEI) Agosto 2007 Disponible en: http://www.inei.gob/ perucifrashtm/inf-dem/cua4.htm. Consultado en Septiembre 2007.

12. Foro Latinoamericano y del Caribe 2007 en VIH/SIDA e ITS: Mujeres conviviendo con el SIDA Informe especial. Disponible en: www.unicef.org/peru/_files/activities/InformeEspecial VIHSIDA.pdf. Consultado en Septiembre 2007.

13. Ministerio de Salud (MINSA). Guía Nacional de Consejería en ITS/VIH y el SIDA. Lima, Perú; 2006. Disponible en: http://www.minsa.gob.pe/portal/ 03Estrategias-Nacionales/03ESN-ITSSIDA/Archibos/GuiaNacionallTS-Dic2006.pdf. Consultado en Septiembre 2007.

14. UNICEF (Lima), Comunidad Internacional de Mujeres Viviendo con VIH/ Sida. Disponible en: http:/ /www.unicef.org/peru/_files/activities/InformeEspecial VIHSIDA.pdf. Consultado en Agosto 2007. 